\title{
A Process-Based Approach in Failure Analysis
}

\author{
George A. Pantazopoulos
}

Submitted: 27 June 2014/Published online: 6 August 2014

(C) ASM International 2014

The aim of this editorial, linked to a previous one [1], is to discuss and share a fundamental thinking process pertaining to the design of failure analysis procedure. The subject of organization of failure investigation is comprehensively presented in the excellent Ref. [2], highlighting also details, examples, and applications from the real world of industry and engineering. The holistic and quality-based approach is an essential element of failure analysis investigation to reach high performance and success to the preset goals, as it is in line to worldwide-adopted quality management systems, such as ISO 9001.

A primary approach based on process-design may serve as a guide or work instruction for failure analysis. The use of flowchart is highly advised in quality systems and process development. A representative and indicative example is shown in Fig. 1, and it has many analogies to the famous Plan-Do-Check-Act (PDCA) cycle of the Quality Guru W. Edwards Deming (1900-1993).

The main steps of such process can be summarized as follows:

1. Submission of the inquiry for failure analysis. In this stage, the inquiry is submitted and registered in order to be properly processed, similarly to a sales inquiry.

2. Background information and review of the work requirements. This is a very critical step for the determination of the needs for resources (human skillsets and equipment), in order to provide a reliable and high quality level service. Legal requirements, safety issues, quality systems certifications, external

G. A. Pantazopoulos $(\bowtie)$

ELKEME Hellenic Research Centre for Metals S.A., 252 Pireaus

Street, 17778 Athens, Greece

e-mail: gpantaz@halcor.vionet.gr audits, etc. should also be considered as significant aspects of the entire work. The identification of the minimum requirements needs very frequently the realization of face-to-face discussions, interviews of the involved personnel, meetings, and teleconferences that stretch the timeline and the incurred costs. The result of the review process may lead to a preliminary cost estimation that is necessary to be presented for management approval.

3. Management approval. As it is always required, a management decision is absolutely mandatory to proceed further to the work planning, considering also the other priorities. In case of an economic offer, customer's official consensus concerning technical specifications, time, and cost is crucial to initiate the work.

4. Allocation of resources and work scheduling. For an effective planning, a team leader must be appointed by the management team, and a start-up meeting among the work team should be planned. Timeline and stages of the failure analysis project, competencies of the involved team members and possibilities for outsource activities, delegation of responsibilities (who does what?)..., are principal issues that are usually discussed. Gantt charts could be the outcome of such meetings, especially for complex failure case studies.

5. Failure analysis investigation. This is in the main core of the process, involving the technical stages of the investigation, starting from sampling or sample selection. The sampling process, together with the proper preservation/maintenance of sensitive samples, is of vital importance for failure analysis. Improper sampling or destruction of sensitive samples may eliminate the chance to obtain conclusive evidence for the root-cause and ruin the entire project. For example, fracture 


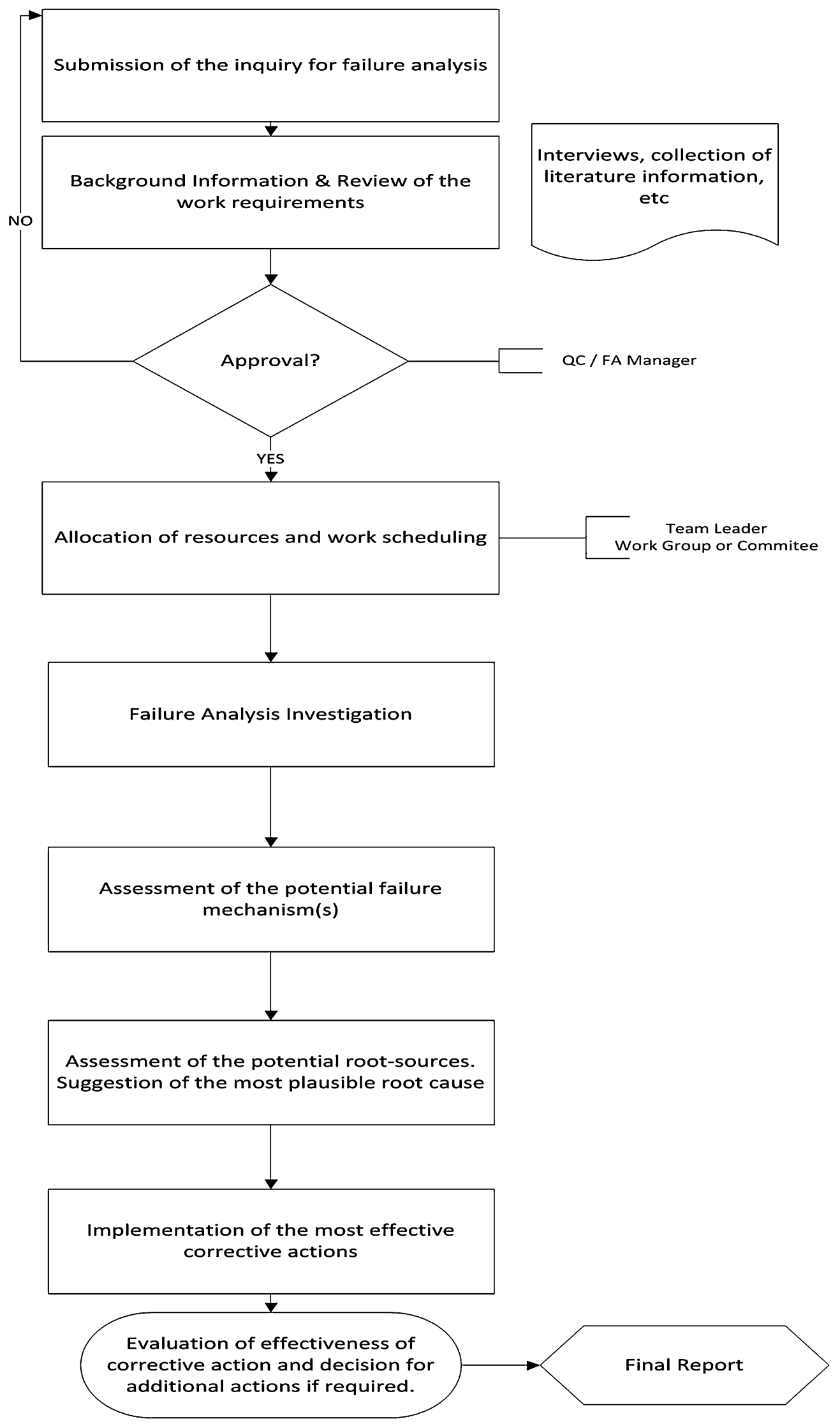

Fig. 1 Flow-chart depicting the general aspects of failure analysis investigation 
surfaces, as very sensitive to oxidation or mechanical damage, unsuitable storage, or careless transportation may cause irreversible damage, obliterating any sign or evidence of failure mechanism(s). Testing and evaluation of specimens using analytical techniques such as non-destructive-testing (NDT), visual inspection, optical microscopy, scanning electron microscopy and microanalysis, hardness/tensile testing, numerical simulation, etc. are very common procedures utilized for failure examination.

6. Assessment of the potential failure mechanism(s). The critical review of the failure analysis ("raw") data and discussion within the corresponding team are required to address the mechanism of failure-i.e., "how" this failure process occur? Via which mechanism or series of mechanisms? In practice and as a simplified view, the principal categories of mechanisms that play a major role to engineering components' operation are:

- Instant mechanisms mechanical overload covering various loading modes (such as tensile, compression, bending, torsion).

- Progressive or time-dependent mechanisms (mostly met in mechanical or machine components' failures) creep, fatigue, corrosion, wear.

- Combined/complex mechanisms combining various individual mechanisms as shown above (e.g., corrosion-fatigue).

The collection and assessment of experimental analysis evidence and logical (common sense) approach will effectively lead to the identification of failure mechanism(s). Critical and objective thinking should prevail in the assessment of the acquired evidence; secondary mechanisms and artifacts should be recognized and neglected. For example, contamination by corrosion substance of the prior fracture surface could provoke confusion regarding the main failure mechanism, leading very frequently to dangerous misinterpretations.

7. Assessment of the potential root-sources. Suggestion of the most plausible root-cause. This stage goes deeper to the understanding of failure process and the interacted causes-addressing the "why" the determined failure mechanism acted? Besides objectivity and impartiality, it requires profound knowledge on the product and process, technical expertise, intelligence, and "talent". Failure analysts should have the ability and analytical skills to reconstruct the failure scene using a backward thinking process, binding the effect with the cause $(s)$. Work team discussions and brainstorming are useful to identify the list of possible root-sources based on logical argumentation. The relationships of "cause-andeffect" could be vividly demonstrated by using quality- organizational tools and techniques, such as the wellknown fishbone diagram (called also as "Ishikawa diagram"). The evaluation of concurrent or interacting causes should also be considered. For example, the stress concentration from geometric design of shaft filet radius together with the poor surface finish will multiply the risk for fatigue failure. Main indicative categories of cause(s) could be considered as follows:

- Manufacturing

- Design/specification

- Testing/evaluation

- Operation/maintenance

- Installation

- Organizational nature

8. Implementation of the most effective corrective actions. Suggestion of corrective actions comes as a natural sequence of root-cause analysis, and it is required in quality management systems for continuous improvement. The possible corrective actions could be also discussed in terms also of the projected efficiency and effectiveness and the most appropriate ones should be selected based on objective arguments, also using time and cost criteria. In the above example, the design revision of the shaft fillet radius will lead to stress calculations that minimize the loading levels below fatigue limit, raising the safety factor and ensuring reliable operation of the component.

9. Evaluation of effectiveness of corrective action and decision for additional actions if required. This stage leads to the confirmation of the problem solving process or to the revision of the action plan in order to achieve the preset target.

10. Final report and closing of the case study. The report summarizes the various intermediate processes followed, the most important findings, and, of course, it is communicated among the involved work team members. This tends to constitute an element of the precious body of knowledge of the failure analysis learning process, documented, checked, and reviewed. Lessons learned could also stand as an individual section or an "epimythion" of the entire essay, reminding us, as members of the society/scientific community, and consistently to failure analysis approach, that we always tend to follow a self-improvement path.

\section{References}

1. G. Pantazopoulos, Failure analysis, quality assurance, and business excellence. J. Fail. Anal. Prev. 13(2), 119-120 (2013)

2. D.P. Dennies, How to Organize and Run a Failure Investigation (ASM International, Materials Park, 2005) 